\title{
UBIQUITOUS REAL-TIME MONITORING OF CRITICAL-CARE PATIENTS IN INTENSIVE CARE UNITS
}

\author{
F. Lamberti, B. Montrucchio \\ Dipartimento di Aut. ed Informatica, Politecnico di Torino, Torino, Italy
}

\begin{abstract}
This paper presents the preliminary results in the development of a framework for ubiquitous monitoring in a Intensive Care Unit environment, which aims at enabling mobile access to real-time bedside monitoring data and to a comprehensive electronic patient's clinical record at any moment in time and from any location, by means of a PDA device capable of secure wireless communications. The main goal of the proposed architecture is to optimise the employment of human resources involved in monitoring activities, while at the same time enhancing the quality of delivered services and improving the level of attention to patients.

Keywords - Telemedicine, ubiquitous computing, Personal Digital Assistant, mobile monitoring, wireless networking
\end{abstract}

\section{INTRODUCTION}

In the last decade, many architectures for remote monitoring have been proposed, which allow remote consultation of patients' clinical information and vital signs, regardless of whether patients are physically located at the hospital site. In most cases, these architectures are based on the use of workstations or personal computers and rely on the availability of fixed network connections via the Hospital Local Area Network, for centralized monitoring within the hospital, or Internet connections, for remote homecare delivery.

The use of assisted monitoring facilities, equipped with hardware of excessive weight or size, limits the usefulness of such solutions in an Intensive Care Unit (ICU) continuous monitoring scenario. In this kind of scenario, alarms have to be immediately notified to skilled medical staff and patients' monitoring data have to be accessed in real time, regardless of whether medical personnel is temporarily outside the ICU or the hospital itself, thus granting absolute mobility (ubiquitous monitoring).

In the past, this issue has been addressed by equipping medical personnel with pagers, which main advantage is to allow a high degree of mobility to ICU staff. However, due to pagers' limited resources (in terms of user interface, computational resources and display capabilities) only transmission of a strictly limited set of alpha-numerical data has been experimented, while consultation and updating of patients' clinical records has not been considered [1].

Recent developments in information and communication technologies, as well as the appearance of truly portable computer devices with greater computing power and display functionalities (in terms of both colors and resolution) and with wireless communication capabilities both in a local and in a wide area environment, allow the deployment of more sophisticated telemedicine applications. In the last years, various PDA and mobile phone-based architectures have been proposed for mobile hospital and home monitoring, remote EPR consultation and applications such as telediagnosis and teleconsulting [2]. In [3,4] a so-called Personal Access and Alert Inferface unit based on a portable device enables medical staff to receive alarm notifications and to access relevant recent vital signs monitoring data concerning monitored patients in Coronary Care Units. In [5] a mobile teleradiology telemedicine unit allows hospital or emergency staff to receive a second-opinion from a specialist participating remotely in a interactive teleconsultation session using a PDA. In $[6,7]$ a consulting physician can access electronic patient records moving inside or outside the hospital by connecting over GSM to a main server using a hand-held companion device based on the Nokia Communicator 9110. In [8] a PDA based telemedicine device enables real-time recording and transmission of ECG data from patients' home or from an emergency site to a remote monitoring site, thus enabling family doctor and emergency staff to receive skilled medical advice.

This paper presents the preliminary results in the development of a framework which aims at ensuring Intensive Care Unit staff mobile access to real-time bedside monitoring data and to a comprehensive electronic patient clinical history, at any moment in time and from any location, by means of a PDA device capable of secure wireless communications, both in a local and in a wide area network environment.

\section{METHODOLOGY}

Even if a number of products for workstation based ICU monitor already exists on the market, we chose to develop a complete prototypal architecture including ICU bedside monitoring network, in order not to depend on a specific commercial solution.

The different components that constitute the mobility enabled monitoring environment that we are designing include the following and are shown in Fig. 1.

\section{A. Artificial bedside monitor}

In a hospital monitoring environment vital signs are acquired on patients at the Intensive Care Unit by means of electrodes and catheters and recorded by means of commercial monitoring systems at the side of each bed in the ICU. However, the development of a monitoring system requires a reproducible and easy-to-control signal source, and patient data does not usually fulfil this requirement. In the experimental phase we have therefore developed a software capable of artificially simulating the behavior of a bedside monitoring device. Moreover, during this initial phase, we could avoid dealing with issues related to the low- 


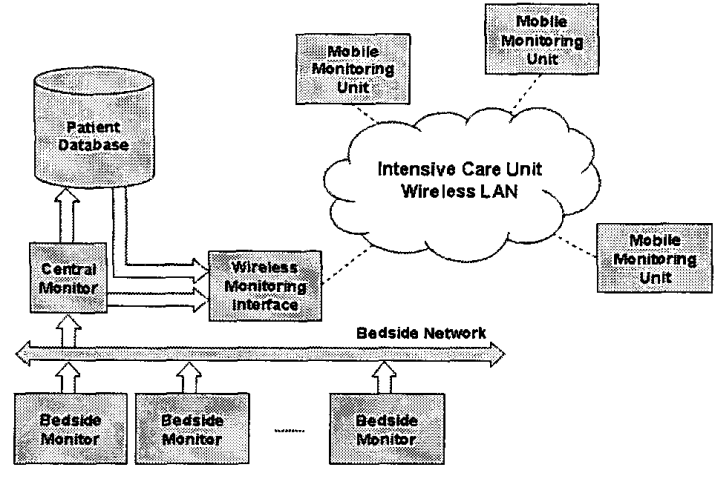

Fig. 1. Intensive Care Unit ubiquitous monitoring overall architecture.

level interface with third-party communication protocols and to the compliancy with medical devices safety standards.

The software, which runs on a desktop PC, is capable of generating artificial waveforms and related alarms for 1-, 2or 3-channel electrocardiogram (ECG), non invasive arterial blood pressure, abdominal respiration or nasal airflow, body temperature, pulmonary and central venous blood pressure, $\mathrm{SpO} 2$ (O2 saturation) and $\mathrm{PCO} 2$ ( $\mathrm{CO} 2$ pressure); waveforms are generated by retrieving medical data from a multichannel annotated reference signal database [9].

\section{B. Central monitor}

Centralized patient monitoring systems installed in the majority of critical care facilities provide the networking of several bedside patient monitors with a central monitoring station (central monitor). The central monitor software which has been deployed to assess the feasibility and effectiveness of the proposed wireless monitoring framework, runs on a desktop PC and receives in real time data and alarms generated by the bedside monitors. Received data are automatically stored in an integrated, patient-based clinical database, compliant with the CEN/TC251 standards. When an alarm condition is detected, remote monitoring functionalities are activated by means of the wireless interface services.

\section{Wireless monitoring interface}

The wireless monitoring interface comprises two modules: the central monitoring interface software (gateway) and the wireless monitoring server. A middleware was defined in order to make the bedside network structure transparent to the remote monitoring subsystem, thus allowing the installation of the proposed mobile monitoring system in heterogeneous hospital infrastructures. Moreover, a suitable software was developed, using an object-oriented methodology, to interface the central monitor with the application that handles communication with mobile terminals. A set of abstract functionalities have been defined and a software implementation has been deployed for the monitoring system proposed in this paper. This approach allows to easily configure the monitoring framework, thus easing the task of designing ad-hoc interfaces for a specific monitoring system.

When the central server detects an alarm condition, the wireless monitoring server notifies the event to the mobile terminals that had previously registered for notification and simultaneously sends recent relevant vital signs. By exploiting middleware services, the wireless monitoring server handles the real-time transmission of monitoring data and the retrieval of patients' clinical history information stored in the main database. The role of the wireless monitoring interface is shown in Fig. 2.

\section{Mobile monitoring unit}

As previously mentioned, when the ICU centralized monitoring system detects an alarm condition, the interface software, which deals with wireless communications, notifies the event to the mobile terminal. Alarm notifications are displayed on the mobile terminal along with the last 30 seconds of waveform data (or of the patient parameter concerned by the alarm). The user can always switch between the data recorded at the time the alarm occurred and current data, which is being recorded and displayed in real time.

The number of waveforms concurrently displayed can be configured and depends on the actual communication channel being used. While the user is moving within the hospital department Wireless Local Area Network, the available bandwidth and mobile terminal resources allow the simultaneous transmission and display of 12 vital signs or parameters at the highest admissible sampling rate. When the user location cannot be reached by the department Wireless LAN, a maximum of three waveforms can be simultaneously displayed, in order to keep data access latency low and to allow uninterrupted continuous real-time monitoring. If the user wishes to view a limited set of signals, or a specific one, the mobile terminal display area is partitioned in order to obtain a display resolution suited for all diagnosis purposes. Fig. 3 illustrates the user interface for mobile vital signs monitoring.

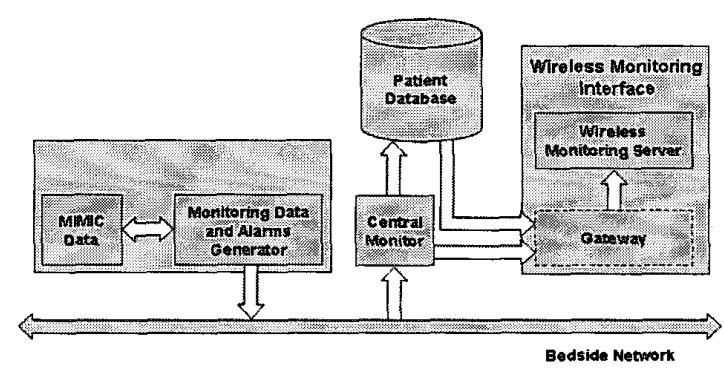

Fig. 2. Detailed view of the system components. 


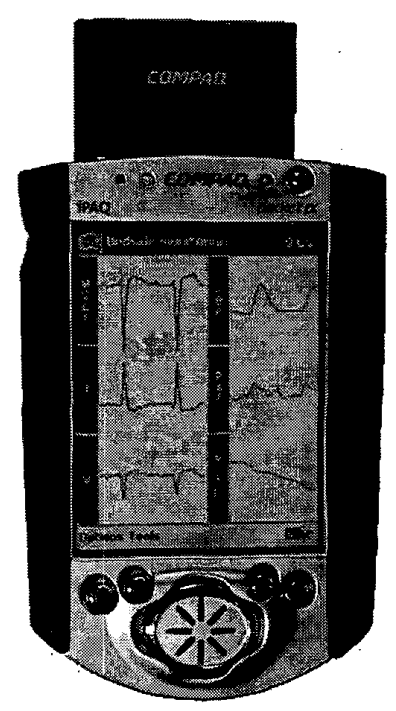

Fig. 3. PDA based interface for real-time vital signs monitoring and alarms notification.

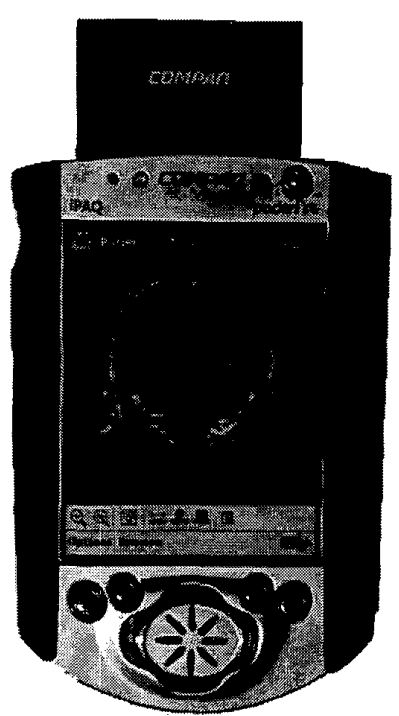

Fig. 4. PDA based user interface for patient's history database access.

Finally, the user can access the main database in which patients' clinical history is stored, including previously monitored signals and parameters, diagnostic images, laboratory analysis and treatments information, as well as admission, demographic and administrative information. In Fig. 4 the user interface enabling database access is shown.

\section{RESULTS}

In this paper a modular architecture for remote patient monitoring has been presented. The main aim of this architecture is to extend Intensive Care Unit monitoring functionalities so that medical staff, equipped with PDAbased portable devices, is able to access in real time all information generated by the bedside monitoring network, on secure wireless communication channels, both from inside and outside the hospital site.

The mobile device allows the user to receive information related to alarms originated from monitored stations and to consult the main database where patients' clinical records, including previously recorded vital signs, medical images, laboratory and treatments information as well as demographic and administrative information, are stored. The proposed solution has been validated by simulating a network of bedside monitors of a real ICU with software modules running on desktop PCs. The approach followed in the development of interface software eases the task of integrating the proposed framework in real application scenarios.

\section{CONCLUSION}

The ICU mobile monitoring architecture proposed in this paper aims to optimise the employment of human resources involved in monitoring activities, while at the same time enhancing the quality of delivered services and improving the level of attention to patients.

As far as future work is concerned, validation of the proposed framework effectiveness into an existing ICU bedside monitoring network is of fundamental importance, and requires the design of the software modules needed to interface the proposed middleware with legacy communication protocols. Moreover, it would be very useful to assess the effectiveness of mobile devices different than PDAs, such as tablet-PCs, which offer wider displays and greater computing power, or $3 \mathrm{G}$ mobile phones which, as soon as they appear on the market, will offer higher bandwidth and thus will be particularly suited for telemonitoring scenarios in a geographical environment. Lastly, but not less important, we consider it necessary to design graphics user interface specifically devoted to both on-line and off-line consultation of huge amount of data on portable devices with limited visualization resources.

\section{REFERENCES}

[1] S. Nelwan, S. Meij, K. Fuchs and T. van Dam, "Ubiquitous access to real-time patient monitoring data," Computers in Cardiology, vol. 24, pp. 271-274, 1997.

[2] C.S. Pattichis, E. Kyriacou, S. Voskarides, M.S. Pattichis, R. Istepanian and C.N. Schizas, "Wireless Telemedicine Systems: An Overview," IEEE Antenna's and Propagation Magazine, vol. 44, pp. 143-153, April 2002.

[3] S. Barro, J. Presedo, D. Castro, M. Fernández-Degaldo, S. Fraga, M. Lama and J. Villa, "Intelligent Telemonitoring 
of critical-care patients," IEEE En. Med. \& Biol. Mag., vol. 18, pp. 80-88, 1999.

[4] J. Presedo, D. Castro, J. Villa, M. Fernández-Degaldo, S. Fraga, M. Lama and S. Barro, "Wireless Interface for Monitored Patients in Coronary Care Unit," in Proc. $22^{\text {nd }}$ Annual EMBS International Conference, Chicago, IL, 1999, pp. 1942-1945.

[5] F. Lamberti, B. Montrucchio and C. Demartini, "Modelling of a GPRS-based mobile telemedicine system for real time monitoring and collaborative diagnosis," in Proc. $4^{\text {th }}$ Int. Workshop on Biosignal Interpretation BSI2002, Como, 2002, pp. 493-496.

[6] J. Reponen, E. Ilkko, L. Jyrkinem, O. Tervonen, J. Niinimaki, V. Karhula and A. Koivula, "Initial Experience with a Wireless Personal Digital Assistant as a Teleradiology Terminal for Reporting Emergency Computerized Tomography Scans," Journal of Telemedicine and Telecare, vol. 6 , pp. 45-49, 2000.

[7] J. Reponen, J. Niinimaki, A. Holopainem, P. Jartti, E. Ilkko, A. Karttunen, T. Kumpulainem, O. Tervonem and E. Paakko, "MOMEDA - A Mobile Smartphone Terminal For DICOM Images and Web-Based.Electronic Patient Data," Journal of Telemedicine and Telecare, vol. 6, 2000, pp. 4549.

[8] F. Lamberti, B. Montrucchio and C. Demartini, "A wireless-based architecture for medical teleconsulting," in Proc. $16^{\text {th }}$ EURASIP Conference BIOSIGNAl 2002, Brno, 2002, pp. 454-456.

[9] G.B. Moody and R.G. Mark, "A Database to Support Development and Evaluation of Intelligent Intensive Care Monitoring," Computers in Cardiology, vol. 23, pp. 657-660, 1996. 